\title{
Special values of the Riemann zeta function via arcsine random variables
}

\author{
Takahiko Fujita
}

\begin{abstract}
In this paper, using arcsine variables, we get a new elementary proof of $\zeta(2)=$ $\pi^{2} / 6$, known as the Basel problem, and the Euler formula. Using exponential variables, we get an Euler-like formula. We can also solve the Basel problem by using Wigner's semicircle law and the Legendre generating function.
\end{abstract}

\section{Introduction}

Let $\zeta(s)=\sum_{j=1}^{\infty} 1 / j^{s}$ be the Riemann zeta function for $\operatorname{Re} s>1$. Many authors have written elementary proofs of $\zeta(2)=\pi^{2} / 6$ (see [3], [6]-[9]). The problem of finding this value is known as the Basel problem (see [2]). In our previous papers [1] and [4], using two independent Cauchy variables, we gave a probabilistic solution of this problem, and obtained the Euler formula of the Riemann zeta function (cf. Theorem 1.1 below).

Let us review these in the new manner of this paper. We start with the following lemma. This is a fundamental lemma, and the proof is easy.

\section{LEMMA 1.1}

Consider two independent random variables $X, Y$ such that $P(X>0)=$ $P(Y>0)=1$ and with density functions $f_{X}(x), f_{Y}(x)$. Then the density function $f_{Y / X}(x)$ of $Y / X$ is given by

$$
f_{Y / X}(x)=\int_{0}^{\infty} f_{X}(u) f_{Y}(u x) u d u .
$$

Proof

For $x>0$,

$$
\begin{aligned}
P\left(\frac{Y}{X}<x\right) & =\iint_{v / u<x} f_{Y}(v) f_{X}(u) d u d v \\
& =\int_{0}^{\infty} f_{X}(u) d u \int_{0}^{u x} f_{Y}(v) d v .
\end{aligned}
$$

Then differentiating both sides with respect to $x$ gives the result.

Kyoto Journal of Mathematics, Vol. 55, No. 3 (2015), 673-686

DOI 10.1215/21562261-3089145, (C) 2015 by Kyoto University

Received October 12, 2011. Revised August 14, 2014. Accepted August 29, 2014.

2010 Mathematics Subject Classification: 11M06, 11M35, 60E05. 
REMARK 1.1

The lemma in [1] was on the density function of the product of two independent random variables. In this paper, we consider the ratio of them.

Let $\mathbb{C}_{1}$ and $\mathbb{C}_{2}$ be independent Cauchy variables; that is, $\mathbb{C}_{1}$ and $\mathbb{C}_{2}$ are independent random variables with $f_{\mathbb{C}_{1}}(x)=f_{\mathbb{C}_{2}}(x)=(1 / \pi)\left(1 /\left(1+x^{2}\right)\right)$. Applying Lemma 1.1 for $X=\left|\mathbb{C}_{1}\right|$ and $Y=\left|\mathbb{C}_{2}\right|$, where $f_{\left|\mathbb{C}_{1}\right|}(x)=f_{\left|\mathbb{C}_{2}\right|}(x)=(2 / \pi)(1 /(1+$ $\left.\left.x^{2}\right)\right) 1_{x>0}$, we get that for $x>0$,

$$
\begin{aligned}
f_{\left|\mathbb{C}_{2}\right| /\left|\mathbb{C}_{1}\right|}(x) & =\frac{4}{\pi^{2}} \int_{0}^{\infty} \frac{1}{\left(1+u^{2}\right)} \frac{1}{\left(1+(u x)^{2}\right)} u d u \\
& =\frac{2}{\pi^{2}} \int_{0}^{\infty} \frac{1}{(1+u)} \frac{1}{\left(1+u x^{2}\right)} d u \\
& =\frac{2}{\pi^{2}} \int_{0}^{\infty}\left(\frac{1}{1+u}-\frac{x^{2}}{1+u x^{2}}\right) \frac{d u}{1-x^{2}} \\
& =\lim _{A \rightarrow \infty} \frac{2}{\pi^{2}} \int_{0}^{A}\left(\frac{1}{1+u}-\frac{x^{2}}{1+u x^{2}}\right) \frac{d u}{1-x^{2}} \\
& =\frac{4}{\pi^{2}} \frac{\log x}{x^{2}-1} .
\end{aligned}
$$

Since $\int_{0}^{\infty} f_{\left|\mathbb{C}_{2}\right| /\left|\mathbb{C}_{1}\right|}(x) d x=1$, we have that

$$
\frac{\pi^{2}}{4}=\int_{0}^{\infty} \frac{\log x}{x^{2}-1} d x .
$$

Here, the right-hand side (RHS) is computed as

$$
\begin{aligned}
& \int_{0}^{1} \frac{\log x}{x^{2}-1} d x+\int_{1}^{\infty} \frac{\log x}{x^{2}-1} d x \\
& \quad=2 \int_{0}^{1} \frac{-\log x}{1-x^{2}} d x=2 \int_{0}^{1}(-\log x) \sum_{k=0}^{\infty} x^{2 k} d x \\
& =2 \sum_{k=0}^{\infty} \int_{0}^{1}(-\log x) x^{2 k} d x=2 \sum_{k=0}^{\infty} \int_{0}^{\infty} u e^{-2 k u} e^{-u} d u \\
& =2 \sum_{k=0}^{\infty} \int_{0}^{\infty} \frac{y}{2 k+1} e^{-y} \frac{d y}{2 k+1}=2 \Gamma(2) \sum_{k=0}^{\infty} \frac{1}{(2 k+1)^{2}} .
\end{aligned}
$$

Thus $\sum_{k=0}^{\infty} 1 /(2 k+1)^{2}=\pi^{2} / 8$. Noting that $\zeta(2)=\sum_{k=1}^{\infty} 1 / k^{2}=\sum_{k=0}^{\infty} 1 /(2 k+$ $1)^{2}+\left(1 / 2^{2}\right) \zeta(2)$, we obtain the desired result, that is,

$$
\zeta(2)=\frac{4}{3} \sum_{k=0}^{\infty} \frac{1}{(2 k+1)^{2}}=\frac{4}{3} \frac{\pi^{2}}{8}=\frac{\pi^{2}}{6} .
$$

This is a probabilistic solution of the Basel problem.

Considering moments of even order of $\log \left|\mathbb{C}_{2}\right| /\left|\mathbb{C}_{1}\right|$, we can prove the Euler formula of the Riemann zeta function. 
LEMMA 1.2

We have that

$$
E\left[\left(\log \frac{\left|\mathbb{C}_{2}\right|}{\left|\mathbb{C}_{1}\right|}\right)^{2 n}\right]=\frac{8}{\pi^{2}} \Gamma(2 n+2)\left(1-\frac{1}{2^{2 n+2}}\right) \zeta(2 n+2), \quad n=0,1,2, \ldots
$$

\section{Proof}

We have that the left-hand side (LHS) is given as

$$
\begin{aligned}
\text { LHS } & =\int_{0}^{\infty}(\log x)^{2 n} f_{\left|\mathbb{C}_{2}\right| /\left|\mathbb{C}_{1}\right|}(x) d x \\
& =\frac{4}{\pi^{2}} \int_{0}^{\infty} \frac{(\log x)^{2 n+1}}{x^{2}-1} d x \\
& =\frac{8}{\pi^{2}} \int_{0}^{1} \frac{(\log x)^{2 n+1}}{x^{2}-1} d x \\
& =\frac{8}{\pi^{2}} \int_{0}^{1}(-\log x)^{2 n+1} \sum_{k=0}^{\infty} x^{2 k} d x=\frac{8}{\pi^{2}} \sum_{k=0}^{\infty} \int_{0}^{1}(-\log x)^{2 n+1} x^{2 k} d x \\
& =\frac{8}{\pi^{2}} \sum_{k=0}^{\infty} \int_{0}^{\infty} u^{2 n+1} e^{-2 k u} e^{-u} d u \\
& =\frac{8}{\pi^{2}} \sum_{k=0}^{\infty} \int_{0}^{\infty}\left(\frac{u}{2 k+1}\right)^{2 n+1} e^{-u} \frac{d u}{2 k+1} \\
& =\frac{8}{\pi^{2}} \Gamma(2 n+2) \sum_{k=0}^{\infty} \frac{1}{(2 k+1)^{2 n+2}} \\
& =\operatorname{RHS}
\end{aligned}
$$

From this lemma, the Euler formula follows.

\section{THEOREM 1.1 (EULER FORMULA)}

We have that

$$
\zeta(2 n+2)=\frac{1}{2}\left(\frac{\pi}{2}\right)^{2 n+2} \frac{1}{1-\frac{1}{2^{2 n+2}}} \frac{A_{n}}{\Gamma(2 n+2)}, \quad n=0,1,2, \ldots,
$$

where the numbers $A_{n}$ are determined by the series development

$$
\frac{1}{\cos ^{2} \theta}=\sum_{n=0}^{\infty} \frac{A_{n}}{(2 n) !} \theta^{2 n} \quad\left(|\theta|<\frac{\pi}{2}\right) .
$$

\section{Proof}

We only have to prove that $E\left[\left|\mathbb{C}_{1}\right|^{\alpha}\right]=1 /\left(\cos \frac{\pi}{2} \alpha\right)(|\alpha|<1)$, because by this, we can easily see that $E\left[e^{\alpha \log \left(\left|\mathbb{C}_{2}\right| /\left|\mathbb{C}_{1}\right|\right)}\right]=1 /\left(\cos ^{2} \frac{\pi}{2} \alpha\right)$, which is equivalent to $E\left[\left(\log \left|\mathbb{C}_{2}\right| /\left|\mathbb{C}_{1}\right|\right)^{2 n}\right]=\left(\frac{\pi}{2}\right)^{2 n} A_{n}$. 
Noting that $\mathbb{C}_{1} \sim N / N^{\prime}$, that is, the distribution of $\mathbb{C}_{1}$ agrees with that of $N / N^{\prime}$, where $N$ and $N^{\prime}$ are two independent standard normal random variables, we get that $\left(\mathbb{C}_{1}\right)^{2} \sim N^{2} /\left(N^{\prime}\right)^{2} \sim \gamma_{1 / 2} / \gamma_{1 / 2}^{\prime}$, where $\gamma_{1 / 2}$ and $\gamma_{1 / 2}^{\prime}$ are two independent gamma variables with parameter $1 / 2$, that is, its density $f_{\gamma_{1 / 2}}(x)=$ $\left(x^{-1 / 2} / \sqrt{\pi}\right) e^{-x}(x>0)$. Then we have that

$$
\begin{aligned}
E\left[\left|\mathbb{C}_{1}\right|^{\alpha}\right] & =E\left[\left(\gamma_{1 / 2}\right)^{\alpha / 2}\right] E\left[\left(\gamma_{1 / 2}\right)^{-\alpha / 2}\right] \\
& =\frac{\Gamma\left(\frac{1}{2}+\frac{\alpha}{2}\right)}{\Gamma\left(\frac{1}{2}\right)} \frac{\Gamma\left(\frac{1}{2}-\frac{\alpha}{2}\right)}{\Gamma\left(\frac{1}{2}\right)} \\
& =\frac{1}{\pi} \frac{\pi}{\sin \pi\left(\frac{1}{2}+\frac{\alpha}{2}\right)} \\
& =\frac{1}{\cos \frac{\pi}{2} \alpha} \quad(|\alpha|<1)
\end{aligned}
$$

where we used the fact that $\Gamma(s) \Gamma(1-s)=\pi /(\sin \pi s)$ and $f_{\gamma_{a}}(x)=\left(x^{a-1} /\right.$ $\Gamma(a)) e^{-x}(x>0), E\left[\left(\gamma_{a}\right)^{b}\right]=\Gamma(a+b) / \Gamma(a)$.

\section{REMARK 1.2}

Since $1 /\left|\mathbb{C}_{1}\right| \sim\left|\mathbb{C}_{1}\right|$, the calculation above is indeed the same as that of $[1]$.

\section{REMARK 1.3}

The number $A_{n}$ is called the tangent number. This name "tangent" comes from (3.1) below. It is known that, for $n \geq 0, A_{n}$ is a positive integer and

$$
A_{n}=(-1)^{n} B_{2 n+2} \frac{2^{2 n+2}\left(2^{2 n+2}-1\right)}{2 n+2} .
$$

Here $B_{n}, n \geq 0$, are the Bernoulli numbers, which are determined by the series development

$$
\frac{x}{e^{x}-1}=\sum_{n=0}^{\infty} \frac{B_{n}}{n !} x^{n} \quad(|x|<2 \pi) .
$$

Substituting the expression of $A_{n}$ into the RHS of the Euler formula in Theorem 1.1, we have

$$
\zeta(2 n)=\frac{(-1)^{n-1}}{2} \frac{(2 \pi)^{2 n}}{(2 n) !} B_{2 n}, \quad n=1,2, \ldots
$$

As the Euler formula of $\zeta(2 n)$, this form is more familiar than ours.

The above is a review of our previous papers [1] and [4].

In this paper, instead of Cauchy, we use arcsine random variables, that is, random variables with density function $2 /\left(\pi \sqrt{1-x^{2}}\right)(0<x<1)$. Then the Basel problem is solved in Section 2, and the Euler formula is derived in Section 3. In Section 4, by using exponential random variables, an Euler-like formula is obtained. The Basel problem is also solved by using Wigner's semicircle random 
variables in Section 5 and the Legendre generating function in Section 6. We note that, in [5], related results about the Hurwitz zeta function are discussed.

\section{Basel problem via arcsine}

Let $X_{1}$ and $X_{2}$ be independent arcsine random variables. For $0<x<1$,

$$
\begin{aligned}
f_{X_{2} / X_{1}}(x) & =\int_{0}^{1} \frac{1}{\sqrt{1-u^{2}} \sqrt{1-u^{2} x^{2}}} \frac{4}{\pi^{2}} u d u=\frac{2}{\pi^{2}} \int_{0}^{1} \frac{d u}{\sqrt{(1-u)\left(1-u x^{2}\right)}} d u \\
& =\frac{2}{\pi^{2}} \int_{0}^{1} \frac{1}{\sqrt{x^{2}\left(\left(u-\frac{1+x^{2}}{2 x^{2}}\right)^{2}-\left(\frac{x^{2}-1}{2 x^{2}}\right)^{2}\right)}} d u \\
& =\frac{2}{\pi^{2} x} \int_{-\frac{1+x^{2}}{2 x^{2}}}^{\frac{x^{2}-1}{2 x^{2}}} \frac{2}{\sqrt{u^{2}-\left(\frac{x^{2}-1}{2 x^{2}}\right)^{2}}} \int_{\frac{1-x^{2}}{2 x^{2}}}^{\frac{1+x^{2}}{2 x^{2}}} \frac{d u}{\sqrt{u^{2}-\left(\frac{x^{2}-1}{2 x^{2}}\right)^{2}}} \\
& =\frac{2}{\pi^{2} x}\left[\log \left(u+\sqrt{u^{2}-\left(\frac{x^{2}-1}{2 x^{2}}\right)^{2}}\right)\right]_{\frac{1-x^{2}}{2 x^{2}}}^{\frac{1+x^{2}}{2 x^{2}}}=\frac{2}{\pi^{2} x} \log \frac{1+x}{1-x}
\end{aligned}
$$

Noting that, for $x>1, P\left(X_{2} / X_{1} \leq x\right)=1-P\left(X_{1} / X_{2} \leq \frac{1}{x}\right)$, we get that

$$
f_{X_{2} / X_{1}}(x)=-f_{X_{2} / X_{1}}\left(\frac{1}{x}\right)\left(\frac{1}{x}\right)^{\prime}=f_{X_{2} / X_{1}}\left(\frac{1}{x}\right) \frac{1}{x^{2}}=\frac{2}{\pi^{2} x} \log \frac{x+1}{x-1} \quad(x>1) .
$$

Putting these together, we have the following proposition.

\section{PROPOSITION 2.1}

We have that

$$
f_{X_{2} / X_{1}}(x)= \begin{cases}\frac{2}{\pi^{2} x} \log \frac{1+x}{1-x} & (0<x<1) \\ \frac{2}{\pi^{2} x} \log \frac{x+1}{x-1} & (1<x<+\infty)\end{cases}
$$

Since

$$
\begin{aligned}
& \int_{0}^{\infty} f_{X_{2} / X_{1}}(x) d x=1 \\
& 2 \int_{0}^{1} f_{X_{2} / X_{1}}(x) d x=\frac{4}{\pi^{2}} \int_{0}^{1} \frac{1}{x} \log \frac{1+x}{1-x} d x,
\end{aligned}
$$

it follows that

$$
\begin{aligned}
\frac{\pi^{2}}{4} & =\int_{0}^{1} \frac{1}{x}\left(x-\frac{x^{2}}{2}+\frac{x^{3}}{3}-\frac{x^{4}}{4}+\cdots-\left(-x-\frac{x^{2}}{2}-\frac{x^{3}}{3}-\frac{x^{4}}{4}-\cdots\right)\right) d x \\
& =2 \int_{0}^{1}\left(1+\frac{x^{2}}{3}+\frac{x^{4}}{5}+\cdots\right) d x \\
& =2 \sum_{j=0}^{\infty} \frac{1}{(2 j+1)^{2}} .
\end{aligned}
$$


Therefore $\sum_{j=0}^{\infty} 1 /(2 j+1)^{2}=\pi^{2} / 8$ gives that $\zeta(2)=\pi^{2} / 6$.

\section{REMARK 2.1}

For $p, q>0$, we denote by $\beta_{p, q}$ a random variable with density $(1 / B(p, q)) x^{p-1}(1-$ $x)^{q-1}(0<x<1)$. This is called a beta random variable with parameters $p$ and $q$. Then $\sqrt{\beta_{1 / 2,1 / 2}}$ is arcsine distributed.

\section{Euler formula via arcsine}

In this section, we derive the Euler formula for $\zeta(2 n)$ by using arcsine variables.

Let $X_{1}$ and $X_{2}$ be independent arcsine random variables. Then, for $\alpha>-1$,

$$
\begin{aligned}
E\left[X_{1}^{\alpha}\right] & =\frac{2}{\pi} \int_{0}^{1} \frac{x^{\alpha}}{\sqrt{1-x^{2}}} d x \\
& =\frac{2}{\pi} \int_{0}^{1} \frac{u^{\frac{\alpha}{2}}}{\sqrt{1-u}} \frac{1}{2} u^{-\frac{1}{2}} d u \\
& =\frac{1}{\pi} \int_{0}^{1} u^{\frac{\alpha-1}{2}}(1-u)^{-\frac{1}{2}} d u \\
& =\frac{1}{\pi} B\left(\frac{1+\alpha}{2}, \frac{1}{2}\right) .
\end{aligned}
$$

For $|\alpha|<1$,

$$
\begin{aligned}
E\left[\left(\frac{X_{2}}{X_{1}}\right)^{\alpha}\right] & =E\left[X_{2}^{\alpha}\right] E\left[X_{1}^{-\alpha}\right] \\
& =\frac{1}{\pi} B\left(\frac{1+\alpha}{2}, \frac{1}{2}\right) \frac{1}{\pi} B\left(\frac{1-\alpha}{2}, \frac{1}{2}\right) \\
& =\frac{1}{\pi^{2}} \frac{\Gamma\left(\frac{1+\alpha}{2}\right) \Gamma\left(\frac{1}{2}\right)}{\Gamma\left(\frac{\alpha}{2}+1\right)} \frac{\Gamma\left(\frac{1-\alpha}{2}\right) \Gamma\left(\frac{1}{2}\right)}{\Gamma\left(1-\frac{\alpha}{2}\right)} \\
& =\frac{1}{\pi} \frac{\pi}{\sin \pi\left(\frac{1+\alpha}{2}\right)} \frac{1}{\frac{\alpha}{2} \Gamma\left(\frac{\alpha}{2}\right) \Gamma\left(1-\frac{\alpha}{2}\right)} \\
& =\frac{1}{\cos \frac{\pi}{2} \alpha} \frac{1}{\frac{\alpha}{2}} \frac{\sin \frac{\pi}{2} \alpha}{\pi}=\frac{\tan \frac{\pi \alpha}{2}}{\frac{\pi \alpha}{2}} .
\end{aligned}
$$

Here we note that

$$
\frac{\tan x}{x}=\sum_{n=0}^{\infty} \frac{A_{n}}{(2 n+1) !} x^{2 n} \quad\left(|x|<\frac{\pi}{2}\right)
$$

by integrating (1.1) from 0 to $x$.

\section{LEMMA 3.1}

We have that

$$
\begin{aligned}
& E\left[\left(\log X_{2}-\log X_{1}\right)^{2 n}\right] \\
& \quad=\frac{8}{\pi^{2}} \Gamma(2 n+1)\left(1-\frac{1}{2^{2 n+2}}\right) \zeta(2 n+2), \quad n=0,1,2, \ldots
\end{aligned}
$$


Proof

We have that

$$
\begin{aligned}
\text { LHS } & =E\left[\left(\log \frac{X_{2}}{X_{1}}\right)^{2 n}\right] \\
& =\int_{0}^{\infty}(\log x)^{2 n} f_{X_{2} / X_{1}}(x) d x=2 \int_{0}^{1}(\log x)^{2 n} \frac{2}{\pi^{2} x} \log \frac{1+x}{1-x} d x \\
& =\frac{4}{\pi^{2}} \int_{0}^{1} 2 \sum_{k=0}^{\infty} \frac{x^{2 k}}{2 k+1}(\log x)^{2 n} d x=\frac{8}{\pi^{2}} \sum_{k=0}^{\infty} \frac{1}{2 k+1} \int_{0}^{1} x^{2 k}(\log x)^{2 n} d x \\
& =\frac{8}{\pi^{2}} \sum_{k=0}^{\infty} \frac{1}{2 k+1} \int_{0}^{\infty} e^{-2 k u} u^{2 n} e^{-u} d u \\
& =\frac{8}{\pi^{2}} \sum_{k=0}^{\infty} \int_{0}^{\infty} e^{-u}\left(\frac{u}{2 k+1}\right)^{2 n} \frac{d u}{(2 k+1)^{2}} \\
& =\frac{8}{\pi^{2}} \Gamma(2 n+1) \sum_{k=0}^{\infty} \frac{1}{(2 k+1)^{2 n+2}} \\
& =\operatorname{RHS} .
\end{aligned}
$$

Comparing (3.1) for $x=\pi \alpha / 2$ with

$$
\begin{aligned}
E\left[\left(\frac{X_{2}}{X_{1}}\right)^{\alpha}\right]= & E\left[e^{\alpha\left(\log X_{2}-\log X_{1}\right)}\right] \\
= & \sum_{n=0}^{\infty} \frac{\alpha^{2 n}}{(2 n) !} E\left[\left(\log X_{2}-\log X_{1}\right)^{2 n}\right] \\
& {\left[\text { because } E\left[\left(\log X_{2}-\log X_{1}\right)^{\text {odd }}\right]=0\right], }
\end{aligned}
$$

we get that

$$
\frac{8}{\pi^{2}} \Gamma(2 n+1)\left(1-\frac{1}{2^{2 n+2}}\right) \zeta(2 n+2)=\frac{A_{n}}{2 n+1}\left(\frac{\pi}{2}\right)^{2 n} .
$$

Thus, the Euler formula is again derived by using arcsine variables.

\section{Euler-like formula via exponential random variables}

In this section, using exponential random variables, we can get an Euler-like formula for special values of the Riemann zeta function.

Let $\mathfrak{e}_{1}$ and $\mathfrak{e}_{2}$ be independent exponential random variables, that is, $\mathfrak{e}_{1}$ and $\mathfrak{e}_{2}$ are independent random variables with $f_{\mathfrak{e}_{1}}(x)=f_{\mathfrak{e}_{2}}(x)=e^{-x}(x>0)$. Then, for $\alpha>-1, E\left[\mathfrak{e}_{1}^{\alpha}\right]=\int_{0}^{\infty} x^{\alpha} e^{-x} d x=\Gamma(\alpha+1)$. For $|\alpha|<1$,

$$
E\left[\left(\frac{\mathfrak{e}_{2}}{\mathfrak{e}_{1}}\right)^{\alpha}\right]=\Gamma(\alpha+1) \Gamma(-\alpha+1)=\alpha \Gamma(\alpha) \Gamma(1-\alpha)=\frac{\pi \alpha}{\sin \pi \alpha} .
$$


Here we put

$$
\frac{x}{\sin x}=\sum_{n=0}^{\infty} \frac{C_{n}}{(2 n) !} x^{2 n} \quad(|x|<\pi) .
$$

\section{LEMMA 4.1}

We have that

$$
E\left[\left(\log \mathfrak{e}_{2}-\log \mathfrak{e}_{1}\right)^{2 n}\right]=2 \Gamma(2 n+1)\left(1-\frac{1}{2^{2 n-1}}\right) \zeta(2 n), \quad n=1,2, \ldots
$$

\section{Proof}

Noting that $f_{\mathfrak{e}_{2} / \mathfrak{e}_{1}}(x)=\left(1 /(1+x)^{2}\right) 1_{x>0}$ (the density of the Pareto distribution), we have that, for $n \geq 1$,

$$
\begin{aligned}
& \text { LHS }=E\left[\left(\log \frac{\mathfrak{e}_{2}}{\mathfrak{e}_{1}}\right)^{2 n}\right] \\
& =\int_{0}^{\infty}(\log x)^{2 n} f_{\mathfrak{e}_{2} / \mathfrak{e}_{1}}(x) d x \\
& =\int_{0}^{\infty}(\log x)^{2 n} \frac{1}{(1+x)^{2}} d x \\
& =2 \int_{0}^{1}(\log x)^{2 n} \frac{1}{(1+x)^{2}} d x \\
& =2 \int_{0}^{1} \sum_{k=1}^{\infty} k(-x)^{k-1}(\log x)^{2 n} d x \\
& =2 \sum_{k=1}^{\infty} k \int_{0}^{1}(-x)^{k-1}(\log x)^{2 n} d x \\
& =2 \sum_{k=1}^{\infty} k(-1)^{k-1} \int_{0}^{\infty} e^{-(k-1) u} u^{2 n} e^{-u} d u \\
& =2 \sum_{k=1}^{\infty} k(-1)^{k-1} \int_{0}^{\infty}\left(\frac{u}{k}\right)^{2 n} e^{-u} \frac{d u}{k} \\
& =2 \sum_{k=1}^{\infty} \frac{(-1)^{k-1}}{k^{2 n}} \Gamma(2 n+1) \\
& =2 \Gamma(2 n+1)\left(\sum_{k=1}^{\infty} \frac{-1}{(2 k)^{2 n}}+\sum_{k=0}^{\infty} \frac{1}{(2 k+1)^{2 n}}\right) \\
& =2 \Gamma(2 n+1)\left(\frac{-1}{2^{2 n}} \zeta(2 n)+\left(1-\frac{1}{2^{2 n}}\right) \zeta(2 n)\right) \\
& =\text { RHS. }
\end{aligned}
$$


Comparing (4.1) for $x=\pi \alpha$ with

$$
\begin{aligned}
E\left[\left(\frac{\mathfrak{e}_{2}}{\mathfrak{e}_{1}}\right)^{\alpha}\right]= & E\left[e^{\alpha\left(\log \mathfrak{e}_{2}-\log \mathfrak{e}_{1}\right)}\right] \\
= & \sum_{n=0}^{\infty} \frac{\alpha^{2 n}}{(2 n) !} E\left[\left(\log \mathfrak{e}_{2}-\log \mathfrak{e}_{1}\right)^{2 n}\right] \\
& {\left[\text { because } E\left[\left(\log \mathfrak{e}_{2}-\log \mathfrak{e}_{1}\right)^{\text {odd }}\right]=0\right], }
\end{aligned}
$$

we get that

$$
2 \Gamma(2 n+1)\left(1-\frac{1}{2^{2 n-1}}\right) \zeta(2 n)=C_{n} \pi^{2 n}, \quad n \geq 1 .
$$

Thus an Euler-like formula is obtained.

\section{THEOREM 4.1}

We have that

$$
\zeta(2 n)=\frac{C_{n} \pi^{2 n}}{2 \Gamma(2 n+1)\left(1-1 / 2^{2 n-1}\right)}, \quad n=1,2, \ldots
$$

\section{REMARK 4.1}

We remark that $C_{n}=(-1)^{n-1} B_{2 n}\left(2^{2 n}-2\right), n \geq 0$. Hence, the formula above is the same as (1.2), so this formula is indeed not 'Euler-like' but 'Euler'.

\section{REMARK 4.2}

A decomposition

$$
\frac{1}{\cos \theta}=\frac{\tan \theta}{\theta} \cdot \frac{\theta}{\sin \theta} \quad\left(|\theta|<\frac{\pi}{2}\right)
$$

is interesting for our discussion. From $\tan \theta / \theta$, we have the Euler formula. From $\theta / \sin \theta$, we also have the Euler formula. From $1 / \cos \theta$, we can get special values $L_{\chi_{4}}(2 n+1)$ of $L_{\chi_{4}}(s)$, where $L_{\chi_{4}}(s)=\sum_{j=0}^{\infty}(-1)^{j} /(2 j+1)^{s}(\operatorname{Re} s>0)$ is an $L$ function associated with the quadratic character $\chi_{4}$. This was already discussed in [1].

\section{REMARK 4.3}

Let $X_{1}, X_{2}, \mathfrak{e}_{1}$, and $\mathfrak{e}_{2}$ be independent random variables such that $X_{i}$ is arcsine distributed and $\mathfrak{e}_{j}$ is exponential distributed. Then $X_{2} \mathfrak{e}_{2}^{1 / 2} /\left(X_{1} \mathfrak{e}_{1}^{1 / 2}\right) \sim\left|\mathbb{C}_{1}\right|$, and

$$
\begin{aligned}
E\left[e^{i \lambda \log \frac{X_{2} e_{2}^{1 / 2}}{X_{1} \mathfrak{e}_{1}^{1 / 2}}}\right] & =E\left[e^{i \lambda \log \left|\mathbb{C}_{1}\right|}\right]=\frac{1}{\cosh \frac{\pi}{2} \lambda}, \\
E\left[e^{i \lambda \log \frac{X_{2}}{X_{1}}}\right] & =\frac{\tanh \frac{\pi}{2} \lambda}{\frac{\pi}{2} \lambda}, \quad E\left[e^{i \lambda \log \left(\frac{\mathfrak{e}_{2}}{\mathfrak{e}_{1}}\right)^{1 / 2}}\right]=\frac{\frac{\pi}{2} \lambda}{\sinh \frac{\pi}{2} \lambda}
\end{aligned}
$$

for $-\infty<\lambda<\infty$. This tells us that a decomposition

$$
\frac{1}{\cosh \frac{\pi}{2} \lambda}=\frac{\tanh \frac{\pi}{2} \lambda}{\frac{\pi}{2} \lambda} \cdot \frac{\frac{\pi}{2} \lambda}{\sinh \frac{\pi}{2} \lambda} \quad(-\infty<\lambda<\infty)
$$


follows from that of $\log \left(X_{2} \mathfrak{e}_{2}^{1 / 2} /\left(X_{1} \mathfrak{e}_{1}^{1 / 2}\right)\right)$ in two independent random variables: $\log \left(X_{2} \mathfrak{e}_{2}^{1 / 2} /\left(X_{1} \mathfrak{e}_{1}^{1 / 2}\right)\right)=\log \left(X_{2} / X_{1}\right)+\log \left(\mathfrak{e}_{2} / \mathfrak{e}_{1}\right)^{1 / 2}$.

We also have a similar interpretation by means of Brownian motion. Let $B_{t}$ be 1-dimensional Brownian motion, let $g_{t}=\sup \left\{s \leq t ; B_{s}=0\right\}$, and let $T_{a}^{*}=$ $\inf \left\{t ;\left|B_{t}\right|=a\right\} \quad(a>0)$. Then $g_{T_{a}^{*}}$ and $T_{a}^{*}-g_{T_{a}^{*}}$ are independent, and

$$
\begin{aligned}
E\left[e^{-\lambda T_{a}^{*}}\right] & =\frac{1}{\cosh \sqrt{2 \lambda} a}, \quad E\left[e^{-\lambda g_{T_{a}^{*}}}\right]=\frac{\tanh \sqrt{2 \lambda} a}{\sqrt{2 \lambda} a}, \\
E\left[e^{-\lambda\left(T_{a}^{*}-g_{T_{a}^{*}}\right)}\right] & =\frac{\sqrt{2 \lambda} a}{\sinh \sqrt{2 \lambda} a} \quad(\lambda>0) .
\end{aligned}
$$

Thus a decomposition

$$
\frac{1}{\cosh \sqrt{2 \lambda} a}=\frac{\tanh \sqrt{2 \lambda} a}{\sqrt{2 \lambda} a} \cdot \frac{\sqrt{2 \lambda} a}{\sinh \sqrt{2 \lambda} a} \quad(\lambda>0)
$$

comes from that of $T_{a}^{*}$ in two independent random variables: $T_{a}^{*}=g_{T_{a}^{*}}+\left(T_{a}^{*}-\right.$ $\left.g_{T_{a}^{*}}\right)$.

\section{Basel problem via Wigner's semicircle law}

A random variable with density function $(4 / \pi) \sqrt{1-x^{2}}(0<x<1)$ is called a Wigner's semicircle random variable. Let $W_{1}$ and $W_{2}$ be independent Wigner's semicircle random variables. For $0 \leq x \leq 1$,

$$
f_{W_{2} / W_{1}}(x)=\int_{0}^{1} \frac{16}{\pi^{2}} \sqrt{1-u^{2}} \sqrt{1-x^{2} u^{2}} u d u=\frac{8}{\pi^{2}} \int_{0}^{1} \sqrt{(1-u)\left(1-x^{2} u\right)} d u .
$$

Then

$$
\begin{gathered}
P\left(W_{2} \leq W_{1}\right)=\frac{1}{2} \\
\| \\
P\left(\frac{W_{2}}{W_{1}} \leq 1\right)=\frac{8}{\pi^{2}} \iint_{0 \leq u, x \leq 1} \sqrt{(1-u)\left(1-x^{2} u\right)} d u d x
\end{gathered}
$$

and thus

$$
\begin{aligned}
\frac{\pi^{2}}{16} & =\iint_{0 \leq u, x \leq 1} \sqrt{1-u}\left(1-\left(1-\sqrt{1-u x^{2}}\right)\right) d u d x \\
& =\frac{2}{3}-\iint_{0 \leq u, x \leq 1} \sqrt{1-u}\left(1-\sqrt{1-u x^{2}}\right) d u d x \\
& =\frac{2}{3}-\iint_{0 \leq u, x \leq 1} \sqrt{1-u} \sum_{k=1}^{\infty} \frac{1}{2^{2 k}(2 k-1)}\left(\begin{array}{c}
2 k \\
k
\end{array}\right) x^{2 k} u^{k} d u d x \\
& =\frac{2}{3}-\sum_{k=1}^{\infty} \frac{1}{2^{2 k}(2 k-1)}\left(\begin{array}{c}
2 k \\
k
\end{array}\right) \frac{1}{2 k+1} B\left(k+1, \frac{3}{2}\right)
\end{aligned}
$$




$$
\begin{aligned}
& =\frac{2}{3}-\sum_{k=1}^{\infty} \frac{1}{2^{2 k}(2 k-1)}\left(\begin{array}{c}
2 k \\
k
\end{array}\right) \frac{1}{2 k+1} \frac{\Gamma(k+1) \Gamma\left(\frac{3}{2}\right)}{\Gamma\left(k+\frac{5}{2}\right)} \\
& =\frac{2}{3}-\sum_{k=1}^{\infty} \frac{1}{2^{2 k}(2 k-1)} \frac{(2 k) !}{k ! k !} \frac{1}{2 k+1} \frac{2^{2 k+2} k !(k+1) !}{(2 k+3) !} \\
& =\frac{2}{3}-2 \sum_{k=1}^{\infty} \frac{1}{(2 k-1)(2 k+1)^{2}(2 k+3)} \\
& \frac{2}{3}-\frac{1}{2} \sum_{k=1}^{\infty}\left(\frac{1}{2 k-1}-\frac{1}{2 k+1}\right)\left(\frac{1}{2 k+1}-\frac{1}{2 k+3}\right) \\
& \frac{2}{3}-\frac{1}{2}\left(-\sum_{k=1}^{\infty} \frac{1}{(2 k+1)^{2}}+\frac{1}{2} \sum_{k=1}^{\infty}\left(\frac{1}{2 k-1}-\frac{1}{2 k+1}\right)\right. \\
& \left.\frac{1}{4} \sum_{k=1}^{\infty}\left(\frac{1}{2 k-1}-\frac{1}{2 k+3}\right)+\frac{1}{2} \sum_{k=1}^{\infty}\left(\frac{1}{2 k+1}-\frac{1}{2 k-3}\right)\right) \\
= & \frac{2}{3}-\frac{1}{2}\left(-\sum_{k=0}^{\infty} \frac{1}{(2 k+1)^{2}}+1+\frac{1}{2}-\frac{1}{3}+\frac{1}{6}\right) \\
= & \frac{1}{2} \sum_{k=0}^{\infty} \frac{1}{(2 k+1)^{2}},
\end{aligned}
$$

where we used that $1-\sqrt{1-x}=\sum_{k=1}^{\infty}\left(1 /\left(2^{2 k}(2 k-1)\right)\right)\left(\begin{array}{c}2 k \\ k\end{array}\right) x^{k}(|x|<1)$. This solves the Basel problem.

\section{REMARK 5.1}

We remark that $E\left[W_{1}^{\alpha}\right]=(2 / \pi) B((\alpha+1) / 2,3 / 2)(\alpha>-1)$. From this, it follows that

$$
E\left[\left(\frac{W_{2}}{W_{1}}\right)^{\alpha}\right]=\frac{1}{1-\frac{\alpha^{2}}{4}} \frac{\tan \frac{\pi \alpha}{2}}{\frac{\pi \alpha}{2}} \quad(|\alpha|<1)
$$

and

$$
W_{1} \sim \sqrt{\beta_{1 / 2,3 / 2}} \quad \text { and } \quad W_{1} \sim U^{1 / 2} X
$$

where $\beta_{1 / 2,3 / 2}$ is a beta random variable with parameters $\frac{1}{2}$ and $\frac{3}{2}$ (cf. Remark 2.1), and $U$ and $X$ are independent random variables such that $U$ is uniformly distributed on $(0,1)$ and $X$ is arcsine distributed.

\section{Two simple derivations of the Basel problem}

In this section, using some double integrals, the first of which is related to the Legendre polynomials, we get other ways to the Basel problem. 


\section{THEOREM 6.1}

We have that

$$
\int_{-1}^{1} d t \int_{-1}^{1} \frac{1}{1-2 x t+t^{2}} d x=\int_{-1}^{1} d x \int_{-1}^{1} \frac{1}{1-2 x t+t^{2}} d t
$$

makes

$$
\zeta(2)=\frac{\pi^{2}}{6}
$$

Proof

For $-1<t<1$,

$$
\begin{aligned}
\int_{-1}^{1} \frac{1}{1-2 x t+t^{2}} d x & =\left[\frac{1}{-2 t} \log \left|1-2 x t+t^{2}\right|\right]_{-1}^{1} \\
& =\frac{1}{-2 t}\left(\log (1-t)^{2}-\log (1+t)^{2}\right) \\
& =\frac{1}{t}(\log (1+t)-\log (1-t)) \\
& =2 \sum_{j=0}^{\infty} \frac{t^{2 j}}{2 j+1} .
\end{aligned}
$$

Then $I=\int_{-1}^{1} d t \int_{-1}^{1} 1 /\left(1-2 x t+t^{2}\right) d x=4 \sum_{k=0}^{\infty} 1 /(2 k+1)^{2}$. Because each term is positive, the interchange of sum and integral is clear. By the Fubini theorem,

$$
\begin{aligned}
I & =\int_{-1}^{1} d x \int_{-1}^{1} \frac{1}{1-2 x t+t^{2}} d t=\int_{-1}^{1} d x \int_{-1}^{1} \frac{1}{(t-x)^{2}+1-x^{2}} d t \\
& =\int_{-1}^{1} d x\left[\frac{1}{\sqrt{1-x^{2}}} \arctan \frac{t-x}{\sqrt{1-x^{2}}}\right]_{-1}^{1} \\
& =\int_{-1}^{1} \frac{1}{\sqrt{1-x^{2}}}\left(\arctan \sqrt{\frac{1-x}{1+x}}+\arctan \sqrt{\frac{1+x}{1-x}}\right) d x \\
& =\frac{\pi}{2} \int_{-1}^{1} \frac{1}{\sqrt{1-x^{2}}} d x=\frac{\pi}{2}[\arcsin x]_{-1}^{1}=\frac{\pi^{2}}{2},
\end{aligned}
$$

where we used the fact that $0<\alpha<\frac{\pi}{2}, 0<\beta<\frac{\pi}{2}$, and $\tan \alpha \tan \beta=1$ give $\alpha+\beta=\frac{\pi}{2}$, because $0<\alpha+\beta<\pi$ and $\cos (\alpha+\beta)=\cos \alpha \cos \beta-\sin \alpha \sin \beta=$ $\cos \alpha \cos \beta(1-\tan \alpha \tan \beta)=0$ imply that $\alpha+\beta=\frac{\pi}{2}$. Thus, $\sum_{k=0}^{\infty} 1 /(2 k+1)^{2}=$ $\pi^{2} / 8$. This solves the Basel problem.

\section{REMARK 6.1}

We can interpret (6.1) the Parseval formula for the Legendre generating function $g(t, x)=1 / \sqrt{1-2 x t+t^{2}}=\sum_{n=0}^{\infty} P_{n}(x) t^{n}$, where $P_{n}(x)=\left(1 / 2^{n} n !\right) \frac{d^{n}}{d x^{n}}\left(x^{2}-\right.$ $1)^{n}$ are the Legendre polynomials, because $\sqrt{n+1 / 2} P_{n}(x)(n=0,1,2, \ldots)$ are C.O.N.S. in $\mathbb{L}_{2}[-1,1]$. 


\section{THEOREM 6.2}

We have that

$$
\int_{-1}^{1} d t \int_{0}^{\infty} \frac{1}{\left(1+x^{2}\right)+t\left(1-x^{2}\right)} d x=\int_{0}^{\infty} d x \int_{-1}^{1} \frac{1}{\left(1+x^{2}\right)+t\left(1-x^{2}\right)} d t
$$

makes

$$
\zeta(2)=\frac{\pi^{2}}{6}
$$

Proof

For $-1<t<1$,

$$
\int_{0}^{\infty} \frac{1}{(1+t)+x^{2}(1-t)} d x=\frac{1}{1-t} \frac{1}{\sqrt{\frac{1+t}{1-t}}}\left[\arctan \frac{x}{\sqrt{\frac{1+t}{1-t}}}\right]_{0}^{\infty}=\frac{\pi}{2} \frac{1}{\sqrt{1-t^{2}}} .
$$

Then LHS $=\int_{-1}^{1}(\pi / 2)\left(1 / \sqrt{1-t^{2}}\right) d t=\pi^{2} / 2$. For $x>0, x \neq 1$,

$$
\int_{-1}^{1} \frac{1}{(1+t)+x^{2}(1-t)} d t=\left[\frac{1}{1-x^{2}} \log \left|\left(1-x^{2}\right) t+\left(x^{2}+1\right)\right|\right]_{-1}^{1}=\frac{\log x^{2}}{x^{2}-1} .
$$

So RHS $=\int_{0}^{\infty}\left(\log x^{2} /\left(x^{2}-1\right)\right) d x=2 \int_{0}^{\infty}\left(\log x /\left(x^{2}-1\right)\right)=4 \sum_{k=0}^{\infty} 1 /(2 k+1)^{2}$. Therefore LHS $=$ RHS gives $\sum_{k=0}^{\infty} 1 /(2 k+1)^{2}=\pi^{2} / 8$, and hence $\zeta(2)=\pi^{2} / 6$.

\section{References}

[1] P. Bourgade, T. Fujita, and M. Yor, Euler's formulae for $\zeta(2 n)$ and products of Cauchy variables, Electron. Comm. Probab. 12 (2007), 73-80. MR 2300217. DOI 10.1214/ECP.v12-1244.

[2] D. Castellanos, The ubiquitous $\pi$, I, Math. Mag. 61 (1988), 67-98. MR 0934824. DOI 10.2307/2690037.

[3] B. R. Choe, An elementary proof of $\sum_{n=1}^{\infty} 1 / n^{2}=\pi^{2} / 6$, Amer. Math. Monthly 94 (1987), 662-663. MR 0935853. DOI 10.2307/2322220.

[4] T. Fujita, A probabilistic approach to special values of the Riemann zeta function, RIMS Kôkyûroku 1590 (2008), 1-9.

[5] T. Fujita and Y. Yano, Special values of the Hurwitz zeta function via generalized Cauchy variables, Kyoto J. Math. 52 (2012), 465-477. MR 2959944.

[6] F. Holme, A simple calculation of $\sum_{k=1}^{\infty} 1 / k^{2}$, Nordisk Mat. Tidskr. 18 (1970), 91-92, 120. MR 0273242.

[7] Y. Matsuoka, An elementary proof of the formula $\sum_{k=1}^{\infty} 1 / k^{2}=\pi^{2} / 6$, Amer. Math. Monthly 68 (1961), 485-487. MR 0123858.

[8] I. Papadimitriou, A simple proof of the formula $\sum_{k=1}^{\infty} k^{-2}=\pi^{2} / 6$, Amer. Math. Monthly 80 (1973), 424-425. MR 0313666.

[9] E. L. Stark, Another proof of the formula $\sum_{k=1}^{\infty} 1 / k^{2}=\pi^{2} / 6$, Amer. Math. Monthly 76 (1969), 552-553. MR 1535429. DOI 10.2307/2316976. 
Department of Industrial and Systems Engineering, Chuo University, 1-13-27, Kasuga, Bunkyo, Tokyo, 112-8551, Japan; rankstatistics@gmail.com 